Propuesta para la creación del Consejo Asesor Del Albergue Universitario

Franco Mella San Román

Revista ES (en y sobre Educación Superior)

Vol.1, N¹-2 / Fecha de publicación: 27/12/2021

e-ISSN: 2718-6539

https://revistas.unlp.edu.ar/ES/index

IIES - Facultad de Odontología

DOI: https://doi.org/10.24215/27186539e029

\title{
Propuesta para la creación del Consejo Asesor Del Albergue Universitario
}

Proposal for the creation of the Advisory Council of the University Hostel

Proposta de criação do Conselho Consultivo do Albergue Universitário

\author{
Abogado Franco Mella San Román \\ Asistente de Dirección de Sumarios - UNLP \\ franco-mella@hotmail.com
}

\section{Resumen}

El presente trabajo, nace ante la necesidad constante de analizar el funcionamiento del Albergue Universitario, como consecuencia de las transformaciones que ha experimentado positivamente desde sus inicios. 
El aumento de la cantidad de plazas disponibles, sumado al objetivo de incrementar hacia el año 2022 al máximo la capacidad del Albergue, nos obliga a pensar en nuevos mecanismos de planificación de esta política de la Universidad y a su vez reformular las herramientas con las que cuenta la Dirección a la hora de solucionar conflictos de índole convivencial que puedan llegar a afectar el normal funcionamiento del Albergue.

Así es que se lleva adelante la propuesta de creación de un espacio de asesoramiento permanente, que llamaremos Consejo Asesor del Albergue Universitario, que sea integrado por diversas áreas de nuestra Universidad y que, pueda ser de gran valor para el desarrollo de la organización, sumando a su integración representación de los estudiantes que residen en él.

\section{Abstract}

This work was born out of the constant need to analyze the operation of the University Hostel, as a consequence of the transformations that it has positively experienced since its inception.

The increase in the number of places available, added to the objective of increasing the hostel's capacity to the maximum by 2022, forces us to think about new planning mechanisms for this University policy and in turn reformulate the tools it has. the Management when solving conflicts of a coexistence nature that may affect the normal operation of the Shelter.

Thus, the proposal to create a permanent advisory space is carried out, which we will call the Advisory Council of the University Hostel, which is made up of various areas of our University and which can be of great value for the development of the organization, adding to its integration representation of the students who reside in it. 


\section{Resumo}

Este trabalho nasceu da necessidade constante de analisar o funcionamento do Albergue Universitário, como consequência das transformações por que passou positivamente desde o seu início.

O aumento do número de vagas disponíveis, somado ao objetivo de aumentar a capacidade do hostel ao máximo até 2022, obriga-nos a pensar em novos mecanismos de planificação desta política universitária e por sua vez reformular os instrumentos de que dispõe. de natureza de coexistência que possa afetar o funcionamento normal do Abrigo.

Assim, é concretizada a proposta de criação de um espaço consultivo permanente, a que chamaremos Conselho Consultivo do Albergue Universitário, que é composto por várias áreas da nossa Universidade e que pode ser de grande valor para o desenvolvimento da organização, acrescentando à sua representação de integração dos alunos que nela residem.

\section{Palabras clave}

Albergue Universitario, Bienestar Universitario, Consejo Asesor, Inclusión, Universidad.

\section{Keywords}

University Shelter, University Welfare, Advisory Council, Inclusion, University.

\section{Palavras chave}


University Hostel, University Welfare, Conselho Consultivo, Inclusion, University.

\section{Caracterización del ámbito de gestión y justificación del tema o problema de interés del TFI definido}

\section{El Albergue Universitario}

El Albergue Universitario se constituye como una de las políticas de inclusión más importantes de nuestra Universidad, abierto en el año 2011, durante la primera gestión del Dr. Arq. Fernando Tauber.

En el Albergue actualmente residen $151^{1}$ estudiantes de distintos puntos del país y de países vecinos. Allí, a los estudiantes se les brindan cuatro comidas diarias, cuentan todos con habitaciones individuales, acceso a internet, salas de computación y espacios de recreación. También cuentan con un profesional en Ciencias de la Educación, quién los acompaña en su desarrollo académico y, asimismo, tienen a disposición un equipo interdisciplinario integrado por Psicólogos y Trabajadores Sociales, que les brindan apoyo en cuestiones relativas a sus relaciones interpersonales y en su adaptación a la vida universitaria.

Actualmente el Albergue Universitario, es gestionado por una Dirección que coordina las diferentes áreas que lo componen. Teniendo bajo su conducción una planta estable de aproximadamente 40 trabajadores, divididos en Área de Cocina, Servicios Generales, Atención Estudiantil, Área Contable, sumadas a

\footnotetext{
${ }^{1}$ Según dictamen de renovación de la beca de Albergue Universitario de la Comisión de Bienestar Universitario y Asuntos Estudiantiles del Honorable Consejo Superior de la Universidad Nacional de La Plata con fecha 5 de diciembre de 2019 y el dictamen de adjudicación a nuevos becarios de idéntica Comisión con fecha 16 de Diciembre de 2019.
} 
las ya mencionadas Áreas de apoyo académico y el equipo interdisciplinario de psicólogos y trabajadores sociales.

El Albergue intenta dar un trato de calidad, brindando todo lo necesario a sus estudiantes a fin de que puedan desenvolverse en óptimas condiciones en el tránsito de su vida universitaria.

El Albergue Universitario establece en su reglamento general ${ }^{2}$ como misión “...promover la igualdad de oportunidades en el ámbito de la educación superior universitaria...", "promoviendo el acceso, permanencia y egreso de estudiantes que deseen comenzar o continuar con sus estudios en la Universidad..."3.

Desde este punto de vista, podemos decir que el Albergue Universitario cumple, desde sus comienzos, con los objetivos propuestos, contando a la fecha, con un total de 30 graduados ${ }^{4}$.

\section{Objetivos}

Objetivo principal

Elaborar una propuesta de planificación estratégica del Albergue Universitario que contemple desde procedimientos de convivencia hasta la consolidación de los procesos y herramientas interdisciplinarios para el análisis e

\footnotetext{
${ }^{2}$ Reglamento aprobado por el Honorable Consejo Superior de la Universidad Nacional de La Plata, en sesión de Noviembre de 2016.

3 Artículo 1. Reglamento General Albergue Universitario

${ }^{4}$ Datos proporcionados en marzo 2020 por el Área de Apoyo Académico del Albergue Universitario - UNLP.
} 
implementación de acciones operativas sobre la vida cotidiana y la estructuración institucional.

\section{Objetivos específicos}

- Desarrollar un espacio de consultas con la participación de funcionarios de áreas vinculadas a la gestión del Albergue y también con estudiantes que residan allí, para indagar acerca de potenciales cambios en los procedimientos de funcionamiento de la convivencia y el futuro de la beca de Albergue Universitario.

- Analizar las normativas de aplicación directa en las actividades del Albergue Universitario, caracterizando los grados de adecuación a las características particulares de la organización.

- Indagar acerca de procesos y normas de instituciones análogas o conexas, en otras instituciones universitarias similares del presente o de otros procesos históricos.

- Construir un cronograma progresivo que atienda a las características institucionales, a las particularidades de los integrantes de la comunidad del Albergue y a las necesidades prioritarias de la agenda presente.

\section{Proyecto de intervención}

Análisis Situacional

El Albergue Universitario se encuentra en la actualidad, dando alojamiento a 151 estudiantes de diversas carreras de la UNLP y con presencia en las 17 Facultades que componen esta Casa de Altos Estudios. A su vez, estos 
provienen de 17 provincias de nuestro país y aloja además a estudiantes de 7 países de Latinoamérica ${ }^{5}$.

Así, el Albergue se establece como un espacio pluricultural, donde coexisten realidades, vivencias y costumbres heterogéneas y, por tanto, complejo.

Alí los becarios se enfrentan a procesos de adaptación vinculados a lo académico (como cualquier estudiante universitario) pero principalmente al entorno social y cultural, que podrá variar en más o en menos respecto de su situación previa al ingreso.

Las costumbres, lo socialmente aceptado en sus comunidades y los valores que son comunes en los lugares de procedencia de cada uno de estos estudiantes, en muchos casos son puestos en crisis en un espacio como el Albergue. Ello es así, toda vez que por un lado podrá haber tantos como estudiantes residentes; y por el otro estarán aquellos valores ponderados por la propia UNLP.

En consecuencia, resulta primordial trabajar en los procesos de adaptación al entorno-albergue. Estos procesos se caracterizan por lo personal, cultural y complejo y deben diferenciarse de aquellos relacionados a lo académico y de aquellos relativos a las cuestiones edilicias y/o de habitación.

Dicha adaptación, requiere especial atención por parte de la administración del Albergue Universitario. La relativización de su importancia, podría poner en jaque los objetivos de la institución, independientemente de que se cumpla con los otros (académicos y de servicios).

El Albergue -como parte integrante de la UNLP- debe ser un espacio social y culturalmente sano en un entorno complejo y con realidades diversas. Mas ello

5 Datos obtenidos del área de apoyo académico del Albergue Universitario - UNLP (Marzo 2020) 
no lo deja exento, sino todo lo contrario, de convivir con problemáticas y conflictos interpersonales y donde es fundamental contar con herramientas aptas para dar respuestas a estos.

En los últimos años el Albergue enfrentó situaciones que pusieron en debate ciertos resortes institucionales y su efectividad en la resolución de conflictos y al mismo tiempo dejaron al desnudo la falta de herramientas formales por parte de la Dirección del Albergue.

Dentro de la ausencia de herramientas formales, podemos mencionar que la normativa vigente resulta insuficiente para el tratamiento de estos conflictos. $\mathrm{Ni}$ en la ordenanza 187/87, ni en reglamento de funcionamiento surgen soluciones eficientes o contemplativas de las diversas problemáticas que se susciten como consecuencia de la convivencia en el Albergue, sin perjuicio de la excepción prevista en su art. 4.

Esta crítica a la normativa vigente surge debido a que los conflictos que se sucedan en el Albergue deberán dar respuestas con especial atención a las características propias del ámbito entre las cuales podemos mencionar: la convivencia constante (donde pueden convivir denunciante y acusado), las relaciones interpersonales más o menos estrechas, el hecho de que el eventual conflicto se sustancia en el lugar de habitación de más de 150 personas, entre otros.

En este sentido es previsible que la comunidad-albergue demande respuestas que cuenten con características tales como: celeridad, legitimidad en la decisión y participación en las mismas de diversas áreas de la comunidad universitaria (incluyendo a actores del propio Albergue), y que al mismo tiempo observe las garantías del derecho defensa y debido proceso. 
Un claro ejemplo de la insuficiencia de normativa disciplinaria, es el que gira en torno a la violencia de género. Para estos casos la UNLP prevé un protocolo específico aprobado por Resolución CS №410/15 el cual puede ser eficiente en el ámbito de la Universidad y sus unidades académicas, pero deviene insuficiente al trasladar su aplicación al ámbito del Albergue Universitario, ya que no prevé medidas específicas.

Nuevamente surge aquí manifiesta la necesidad de dar respuestas claras, a tiempo y efectivas. Recordemos que, de conformidad con el desarrollo del presente trabajo, los conflictos que se suscitan en el albergue presentan dos características destacables: potencialidad y expansión.

Para robustecer entonces la legitimidad de las decisiones tomadas ante estos conflictos, es necesario que ellas revelen transparencia en las mismas. Esa transparencia podría ser aportada por un Consejo Asesor donde se encuentren representados todos los actores de la comunidad-albergue y funcionarios de las distintas áreas de la Universidad que tengan incidencia y relación con la temática6.

Proyecto de Intervención

Como resultado de dar inicio a la propuesta es necesario esquematizar en una primera instancia los objetivos a alcanzar:

- Mejorar los mecanismos de respuesta institucionales a los conflictos interpersonales que surgen en el seno del Albergue.

- Establecer espacios de asesoramiento y asistencia permanente a la Dirección del Albergue Universitario, siendo a su vez un espacio donde se contribuya al trazado de políticas y objetivos institucionales.

\footnotetext{
${ }^{6}$ Noticia Diario El Día 11/05/2011 https://www.eldia.com/nota/2012-5-11-conflicto-poruna-expulsion-en-el-albergue-universitario
} 
- Propiciar el mejoramiento de las herramientas comunicacionales existentes entre la dirección del Albergue y los becarios, tornándolas más eficaces e idóneas.

La propuesta se centra en la creación de un Consejo Asesor del Albergue Universitario, que como tal tenga a cargo, con una mirada multidisciplinaria, por un lado, el tratamiento de las diversas problemáticas que puedan presentarse en el Albergue y, por otro, la planificación a largo plazo de la Institución.

¿Por qué un Consejo Asesor?

Las características del Albergue que se desarrollaron a lo largo del presente trabajo, demandan una labor planificada, acompañada por la colaboración de todos los actores de la comunidad universitaria.

A su vez, al tratarse del destino de una dependencia de la UNLP, no parece poco razonable pretender replicar el modelo colegiado y con pluralidad de voces que caracteriza a la universidad argentina, consagrado a partir de la reforma universitaria de 1918. Salvando las distancias con los órganos de cogobierno de universidades y facultades, se pretende la creación de un espacio de asesoramiento de una organización interna de la universidad.

Por otra parte, y como expresa el Dr. Arquitecto Fernando Tauber ${ }^{7}$ en su tesis de Doctorado, es sustancial la idea de la planificación estratégica participativa. Los procesos bajo esa concepción consolidan la posibilidad de múltiples visiones de la realidad que permiten pensar el futuro de las instituciones de forma integral y a través del consenso de sus integrantes.

7 Tauber, Fernando (2008). Tesis de Doctorado "La comunicación en la planificación y gestión para el desarrollo de las instituciones universitarias públicas argentinas: el caso de la Universidad Nacional de La Plata en el trienio junio 2004-mayo 2007" 
El Consejo Asesor del Albergue Universitario (CAAU)

Se propone la creación de un espacio consultivo y de deliberación de las políticas a trazar hacia el interior de la organización, que planifique el futuro de la misma desde una mirada compleja y participativa.

Un espacio que pueda aportar apoyo, acompañamiento y conformidad a las decisiones que eventualmente ejecutará la Dirección del Albergue Universitario, generando de este modo los mayores consensos posibles para legitimar el actuar de quienes deben administrar esta dependencia.

Es necesario que el Consejo Asesor funcione desde una óptica multidisciplinaria, para lo cual se propone que el espacio sea integrado de forma permanente por representantes de áreas que tengan una incidencia relevante en el funcionamiento diario de la institución o que, sin tenerlo, cuenten con herramientas profesionales que se requieran para la planificación y la resolución de conflictos del Albergue.

Composición del Consejo Asesor del Albergue Universitario

- $\quad$ Director del Albergue Universitario

- $\quad$ Prosecretaría de Bienestar Universitario

- Prosecretaria de Derechos Humanos

- Miembro del Equipo Interdisciplinario del Albergue

- Dirección de Políticas de Salud Estudiantil de la Prosecretaría de bienestar Universitario

- $\quad$ Estudiante del Albergue

- Miembro de la Comisión de Bienestar Universitario del Consejo Superior

Funciones del Consejo Asesor del Albergue Universitario

La formulación de tareas que tendrá a cargo el Consejo Asesor que aquí se realiza, es de carácter orientativo y probablemente al momento de llevar 
adelante la propuesta e incluso con la intervención de los demás espacios podrá perfeccionarse a partir de sugerencias que la enriquezcan.

a) Desarrollo del Reglamento interno de funcionamiento del Albergue Universitario.

b) Asesoramiento a la Dirección del Albergue Universitario en conflictos que pongan en riesgo el normal funcionamiento, los valores, fines y objetivos de la institución.

c) Proponer políticas de prevención a las problemáticas convivenciales

d) Dar impulso y promover la confección de Acuerdos de Convivencia de los becarios de la Institución.

e) Llevar adelante actividades dentro del Albergue que fomenten los valores rectores de nuestra Universidad y promuevan la resolución pacífica de conflictos.

f) Trazar acuerdos con las Secretarías de Bienestar o de Asuntos Estudiantiles, de las 17 facultades que componen nuestra universidad con el objetivo de generar espacios de cooperación con las unidades académicas, para poder realizar un seguimiento más eficiente de los estudiantes del Albergue.

g) Generar herramientas de difusión de las actuaciones del Consejo Asesor, con el objetivo de dar publicidad a sus decisiones, la planificación y objetivos institucionales.

\section{Reflexiones finales}

Este trabajo fue realizado con el objetivo de desarrollar una propuesta para el Albergue Universitario, mediante la cual se puedan hacer frente a los nuevos 
desafíos que presenta la institución, sin dejar de cumplir con los objetivos y misiones presentes en el reglamento general del Albergue.

Nuestra universidad ha demostrado con creces su compromiso inagotable con la educación pública y con el objetivo de acercarle a todas las personas que deseen estudiar una carrera universitaria las herramientas necesarias, evitando que cuestiones económicas, sociales o culturales determinen lo contrario.

En particular la propuesta de la creación del Consejo Asesor busca brindar la posibilidad de contar con un espacio de reflexión, de análisis y planificación colectiva, con el norte puesto en que el Albergue Universitario prospere y en generar un espacio propicio para el cumplimiento de los objetivos de crecimiento planificados.

Iniciamos este trabajo realizando un relato de la historia de nuestra universidad y la magnitud de la tarea que se lleva hacia el interior de la misma. Se efectuó un breve recuento de la tarea que realiza nuestra Universidad en materia de Bienestar Universitario y la responsabilidad de seguir estando a la altura de tal proceso.

Explicamos el crecimiento que ha vivido en estos años el Albergue Universitario y cómo su realidad se fue complejizando a la vez que crecía estructural y cuantitativamente. Eso llevó a que, a lo largo de su historia, haya registrado diversas transformaciones de distinta índole, lo cual significó que ciertas herramientas que resultaban suficientes, hoy ya no lo sean.

Como consecuencia de eso y con el objetivo de dar respuestas a estos incipientes desafíos que presenta la nueva realidad del Albergue, se propone trabajar sobre la idea de un Consejo Asesor. Se analiza la experiencia de los Consejos Sociales dentro de las Universidades para conocer experiencias exitosas de espacios de asesoramiento dentro de las universidades y la de los 
Consejos Escolares de Convivencia, en el ámbito de la educación de nivel medio, con la idea de conocer cómo funciona una institución con fines y objetivos que podrían llegar a ser similares -a los aquí planteados- en torno a la regulación de acuerdos de convivencia, resolución de conflictos y planificación institucional con la participación de miembros estudiantes de la comunidad.

A lo largo del proyecto se realiza el desarrollo de las funciones que tendrá a cargo el Consejo Asesor, como así también las etapas para su puesta en funcionamiento

La composición del Consejo Asesor, marca sin duda alguna, el camino que a nuestro juicio se debería recorrer, donde como lo ha demostrado nuestra Universidad, la idea de la planificación por medio de la pluralidad de voces y la búsqueda de consensos, ha sido una marca registrada del modelo reformista de la Universidad Argentina.

\section{Bibliografía}

- Ana García de Fanelli (2016), Educación superior en Iberoamérica Informe Nacional- Argentina, https://cinda.cl/wpcontent/uploads/2019/01/educacion-superior-en-iberoamerica-informe2016-informe-nacional-argentina.pdf

- Clark Rafael I. (2017), Autonomía universitaria y control judicial. Contornos del recurso directo del art. 32 de la Ley de Educación Superior. Edit. Ad-Hoc

- Consejo Escolar de Convivencia (2010), cuadernillo para las escuelas. Ministerio de Educación, Presidencia de la Nación.

- Consejo Interuniversitario Nacional. (2010). La universidad pública en el año

del bicentenario. 
https://www.unc.edu.ar/sites/default/files/2unc seu universidades bicent enario cin.pdf

- Díaz Díaz, Sandra Janet - López Duque, Eliana Patricia (2009), Tesis Representaciones sociales sobre bienestar universitario de los representantes estudiantiles de la Universidad del Quindío, http://bibliotecavirtual.clacso.org.ar/Colombia/alianza-cindeumz/20130319045631/Tesiseliana.pdf

- Diccionario Real Academia Española (2020) https://www.rae.es/

- Doberti, J. (2014), La organización académica en la universidad, el caso de la UBA, Buenos Aires, Universidad de Buenos Aires, Facultad de Ciencias Económicas

- Émile Durkheim (1893) División del Trabajo Social.

- Fornasari, M. (2016). Consejos de Convivencia Escolar. Ética y democracia educativa. Córdoba: Ferreyra Editor

- Huertas Franco (1994) El método pes, entrevista a Carlos Matus. Edit. Angular.

- Jefferson Thomas (2014). Escritos Políticos, Declaración de Independencia, Autobiografía, Epistolario, primera edición, Edit. Tecnos, Madrid.

- Maier Julio B.J. (2004) Derecho Procesal Penal: Fundamentos, 2da edición, Buenos Aires, Edit. Editores del Puerto.

- Manuel Maria Diez (1981), Manual de Derecho Administrativo, 2da edición. editorial PlusUltra

- Miguel S. Marienhoff (1965), Tratado de Derecho Administrativo. Edit. Abeledo Perrot 
- Ministerio de Educación. Consejo Federal de Educación (2009). Resolución CFE Nro. 93/09, Orientaciones para la organización pedagógica e institucional de la Educación Secundaria Obligatoria. https://www.argentina.gob.ar/sites/default/files/93-09-anexo5900b6d3bab8e.pdf

- Miriam M. Ivanega (2012). El derecho de defensa en el procedimiento disciplinario. Buenos Aires. Rap Digital.

- Néstor Cecchi (2013) - El compromiso social de la Universidad Latinoamericana del Siglo XXI : entre el debate y la acción - 2a ed. Buenos Aires : IEC - CONADU.

- Pedro Nuñez (2017) Las políticas de convivencia escolar en el nivel secundario argentino: tradiciones e innovaciones en la regulación de las interacciones escolares, CONICET-FLACSO-UBA, http://www.congresoalacip2017.org/arquivo/downloadpublic2?q=YToyOn tzOjY6InBhcmFtcyl7czozNToiYToxOntzOjEwOiJJRF9BUIFVSVZPljtzOj Q6ljixODliO30iO3M6MToiaCI7czozMjoiNGNhYTcyYzExMmEzYTg3M2U 2NTBkMzM4NTg5ZDc0NmMiO30\%3D

- RedBien (2008) El Bienestar Estudiantil en Argentina Líneas metodológicas y modelos de intervención., Documento presentado en Cali, Colombia, en el Congreso de ASCUM. http://www.redbien.edu.ar/upload/Cali 2008.pdf

- Repetto Alfredo L. (2014) Procedimiento Administrativo Disciplinario: El Sumario, Segunda edición Buenos Aires: Cáthedra Jurídica.

- Secretaría de Políticas Universitarias Presidencia de La Nación Sistemas consulta de estadísticas universitarias. 
- Sousa, E. (2014). El Consejo Escolar: La inclusión de los estudiantes. VIII Jornadas de Sociología de la UNLP, 3 al 5 de diciembre de 2014, Ensenada, Argentina. En Memoria Académica.

- Tauber Fernando (2018). Pensar la universidad proyecto institucional de la Universidad Nacional de La Plata 2018-2022.

- Tauber, Fernando (2008). Tesis de Doctorado "La comunicación en la planificación y gestión para el desarrollo de las instituciones universitarias públicas argentinas: el caso de la Universidad Nacional de La Plata en el trienio junio 2004-mayo 2007".

- Tawil, Guido Santiago (2010), Procedimiento Administrativo. 1era edición - Buenos Aires; Abeledo Perrot.

- Ziulu Adolfo G. (2014), Derecho Constitucional. primera edición, Buenos Aires. Edit Abeledo Perrot. 